\title{
Layout-Aware Yield Prediction of Photonic Circuits
}

\author{
Wim Bogaerts, Umar Khan and Yufei Xing \\ Ghent University - imec, Photonic Research Group, Department of Information Technology, Gent, Belgium. \\ Center of Nano and Biophotonics, Ghent University, Belgium. \\ Email: wim.bogaerts@ugent.be
}

\begin{abstract}
We demonstrate yield prediction of silicon wavelength filter circuits using layout-aware Monte-Carlo circuit simulations. Maps of wafer and die-level variability of width and thickness are projected onto circuit layout and translated into changes of circuit model parameters. We apply this onto MachZehnder lattice filters to study yield for different filter orders.
\end{abstract}

\section{INTRODUCTION}

Silicon photonics enables large-scale integration of photonic circuits thanks to high index contrast submicrometer waveguides. But this high contrast also all components sensitive to nanometer-scale geometry variations. This in turn affects the circuit performance, especially as circuits become larger. Efficient yield prediction in the design stage is an essential requirement for scaling up silicon photonic circuits [1].

This is especially needed for wavelength filters, as these are very sensitive to phase variations and coupling variations . Design processes for wavelength filters are well known, but an accurate prediction of their yield in the presence of fabrication variations is not trivial. Monte-Carlo methods with random variations give an idea of the sensitivity of the circuit, but this is not a realistic representation of actual fabrication variation. For instance, a silicon wire waveguide is most sensitive to the line width and the waveguide thickness, and these are affected by very different processes during the fabrication, with a different distribution over the wafer. Also, variations within the circuit are correlated: elements closer together are more likely to be matched than elements further apart. A good yield prediction therefore depends on the correct mapping of the various sources of variability at die or wafer level caused by the fabrication as well as the design (e.g. pattern density) [3], [4]. Random Monte-Carlo methods, but also faster methods based on Polynomial Chaos expansion [2] can not easily take these different mappings into account.

The Monte-Carlo method can be enhanced by incorporating the spatial information in the form of wafer maps [3], [4]. We have built such a circuit-level variability analysis tool for yield analysis with variable fabrication processes. We separate the concerns of the compact model building and the mapping with the process statistics. When component designers (or fabs) build a compact circuit model for a building block, they are not aware of the larger circuit context which a designer will encounter in his specific design. However, a component designer can still simulate or measure the sensitivity of the component to a number of fabrication effects, such as linewidth or thickness variation. This sensitivity or correlation can be embedded

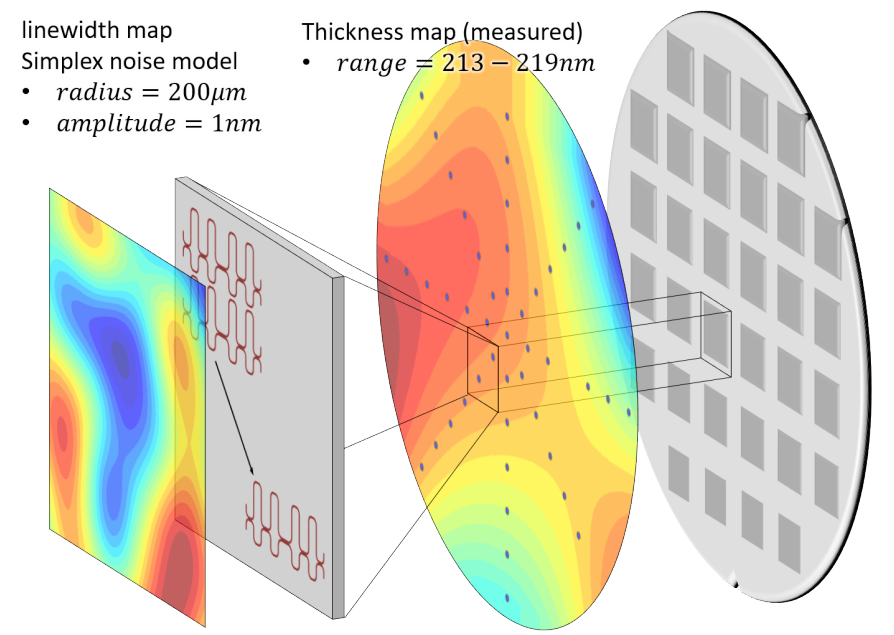

Fig. 1. Maps of processing-related parameters such as deviations in linewidth and thickness are projected onto the circuits in the actual layout.

in the model of the component, or supplied as an additional lookup table. In parallel, fabrication conditions are generally monitored through statistical process control based on inline measurement of test structures (e.g. SEM measurements, ellipsometry) or extracted from measurements on devices. This results in different wafer maps for all important parameters that affect a component's behavior.

The models with sensitivity and the wafer maps are combined into a Monte-Carlo simulation using different locations on a wafer (different dies and within the same die) or on different wafers. This results in a yield prediction that takes into account the actual layout and distribution of the circuit on the chip.

\section{IMPLEMENTATION}

We implemented the variability engine (VE) onto the commercial circuit simulator Caphe by Luceda Photonics [5], which is integrated in the design framework IPKISS with access to the circuit's parametric layout. Caphe allows the implementation of efficient full-custom models in Python, both in time domain and frequency-domain. We extended the circuit models with sensitivity data that can be supplied by the user or the fab, expressing how every model parameter is sensitive to fabrication variables. There is no need for expensive interpolated models: only the sensitivity is needed.

Wafer maps for variables such as thickness and line width can be generated from measurements or from models. Such 


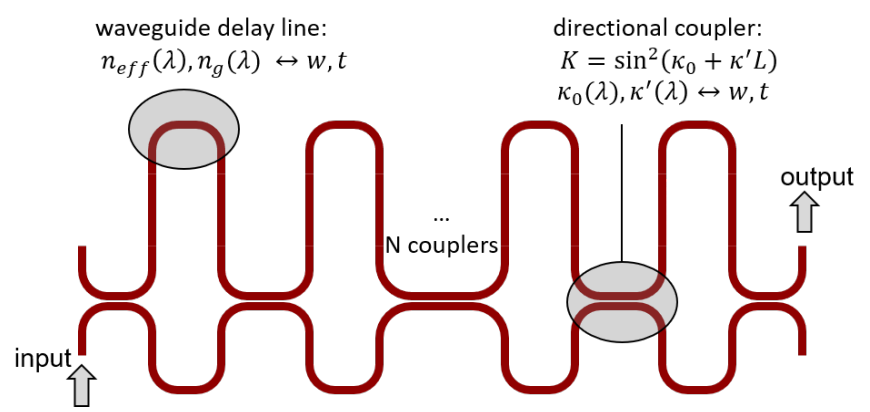

Fig. 2. Mach-Zehnder lattice filter and the sensitivities in the individual building blocks (waveguides and directional couplers)

mapping functions can be provided as black boxes by the fabs, so the actual maps can be used without giving circuit-level designers an actual knowledge of the fab processes.

\section{EXAMPLE}

As an example we used Caphe-VE to evaluate multistage Mach-Zehnder lattice filters consisting of directional couplers and delay lines, shown in Fig. 2, for a SOI platform with $450 \mathrm{~nm} \times 220 \mathrm{~nm}$ wire waveguides. These elements are both very sensitive to linewidth and thickness variation. The dispersive models for the waveguides and the directional coupler building blocks are synthesized from simulations and measurements.

Linewidth and thickness changes will affect the coupling coefficients of the directional coupler as well as the phase delay, which in turn will affect the wavelength peak position, the uniformity of the pass band, and the crosstalk of the filter. For different positions on the die/wafer we expect different filter responses. But an MZI lattice filter consists of multiple stages, and all of these must be matched properly for the filter to work. Therefore, the linewidth and thickness are sampled

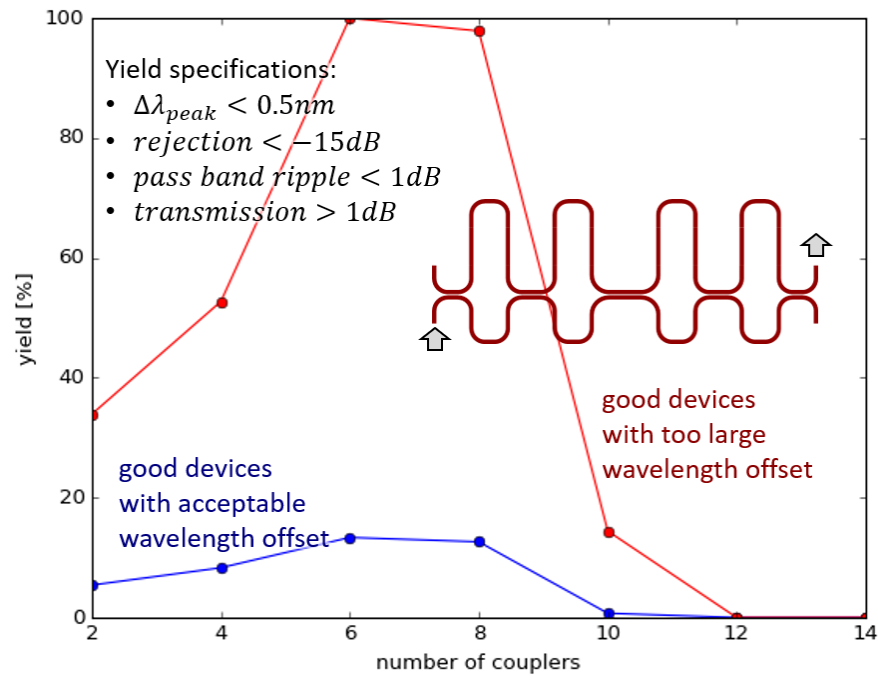

Fig. 3. Overall predicted yield on the wafer for the MZI lattice filter with different number of stages.

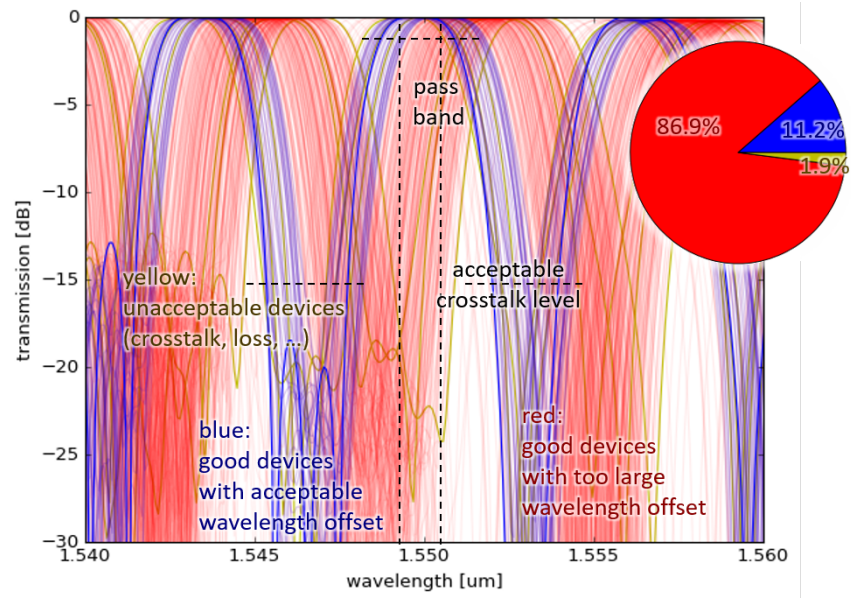

Fig. 4. 280 transmission curves for the 8-coupler filter over the wafer. Blue curves indicate good filters, while red curves indicate good filters but with an unacceptably large wavelength offset.

on several hundred positions within each filter circuit, so the variations within the circuit are also properly modelled. Each component is aware of its own sampling locations.

When we increase the number of stages in the filter, we see the impact of variability on the yield, shown in Fig. 3 . For perfect technology the quality of the filter should improve with the addition of stages, which we see for low number of stages. But because of variability, we then see a deterioration for higher numbers when the matching of stages and the precise control of the coupling becomes critical. While this is not a new insight, it is usually very difficult to accurately and quantitatively model this effect, and optimize a filter for performance in actual process conditions. When looking at the result for 8 couplers in Fig. 4, we see that the absolute position of the filter peak dominates the yield.

\section{CONCLUSION}

We predict the yield of filter circuits in the presence of fabrication variations based on spatially correlated maps of linewidth and thickness. We applied this to MZI lattice filters of different order.

\section{ACKNOWLEDGMENT}

Part of this work is supported by the Flemish Research Foundation (FWO-Vlaanderen) with grant G013815N. Part of this work is supported by the Flemish Agency for Innovation and Entrepreneurship (VLAIO) with the MEPIC project.

\section{REFERENCES}

[1] W. Bogaerts and L. Chrostowski, Laser and Photonics Reviews, vol. 1700237, pp. 1-29, 2018.

[2] T.-W. Weng, D. Melati, A. Melloni, and L. Daniel, Nanophotonics, vol. 6, no. 1, pp. 299-308, 2017.

[3] Z. Lu, J. Jhoja, J. Klein, X. Wang, A. Liu, J. Flueckiger, J. Pond, and L. Chrostowski, Optics Express, vol. 25, no. 9, p. 9712, 2017.

[4] J. Pond, J. Klein, J. Flückiger, X. Wang, Z. Lu, J. Jhoja, and L. Chrostowski, p. 102420S, 2017.

[5] M. Fiers, T. Van Vaerenbergh, K. Caluwaerts, D. Vande Ginste, B. Schrauwen, J. Dambre, and P. Bienstman, Journal of the Optical Society of America B, vol. 29, no. 5, p. 896, 2012. 